\title{
Le Fouineur : un programme d'aide à la réussite scolaire de la ville de Repentigny
}

The Fouineur: A Programme that Supports Academic Success in the City of Repentigny

\section{El Fouineur: un programa de ayuda al éxito escolar de la ciudad de Repentigny}

\section{Chantal Brodeur}

Volume 57, numéro 2, avril-juin 2011

Les services d'information pour les jeunes : actualités et perspectives

URI : https://id.erudit.org/iderudit/1028876ar

DOI : https://doi.org/10.7202/1028876ar

Aller au sommaire du numéro

Éditeur(s)

Association pour l'avancement des sciences et des techniques de la documentation (ASTED)

ISSN

0315-2340 (imprimé)

2291-8949 (numérique)

Découvrir la revue

Citer cet article

Brodeur, C. (2011). Le Fouineur : un programme d'aide à la réussite scolaire de la ville de Repentigny. Documentation et bibliothèques, 57(2), 111-119.

https://doi.org/10.7202/1028876ar
Résumé de l'article

Se basant sur le lien indissociable entre accès aux livres et réussite scolaire, cet article présente la création d'un programme d'aide à la réussite scolaire mis sur pied par la Bibliothèque municipale de Repentigny, en banlieue de Montréal. Il rend compte du projet-pilote mené en 2006-2007 auprès de deux écoles primaires situées dans des quartiers défavorisés et décrit les résultats obtenus au fil des ans. Pour donner le goût des devoirs aux jeunes, Le Fouineur mise sur l'utilisation des ressources documentaires de qualité et sur la disponibilité de spécialistes à la bibliothèque municipale. Dix mille enfants ont participé aux ateliers du Fouineur jusqu'à ce jour.
Tous droits réservés ( $)$ Association pour l'avancement des sciences et des techniques de la documentation (ASTED), 2011
Ce document est protégé par la loi sur le droit d'auteur. L'utilisation des services d'Érudit (y compris la reproduction) est assujettie à sa politique d'utilisation que vous pouvez consulter en ligne.

https://apropos.erudit.org/fr/usagers/politique-dutilisation/ 


\title{
Le Fouineur : un programme d'aide à la réussite scolaire de la ville de Repentigny
}

\author{
CHANTAL BRODEUR \\ Chef de division Bibliothèques \\ Ville de Repentigny \\ brodeurc@ville.repentigny.qc.ca
}

\section{RÉSUMÉ | ABSTRACTS | RESUMEN}

Se basant sur le lien indissociable entre accès aux livres et réussite scolaire, cet article présente la création d'un programme d'aide à la réussite scolaire mis sur pied par la Bibliothèque municipale de Repentigny, en banlieue de Montréal. Il rend compte du projetpilote mené en 2006-2007 auprès de deux écoles primaires situées dans des quartiers défavorisés et décrit les résultats obtenus au fil des ans. Pour donner le goût des devoirs aux jeunes, Le Fouineur mise sur l'utilisation des ressources documentaires de qualité et sur la disponibilité de spécialistes à la bibliothèque municipale. Dix mille enfants ont participé aux ateliers du Fouineur jusqu'à ce jour.

The Fouineur: A Programme that Supports Academic Success in the City of Repentigny

Based on the natural link between access to books and academic success, this article describes the creation of a programme designed to support academic success; it was established by the public library of Repentigny, a suburb of Montréal. The article describes the pilot project set up in 2006-2007 with the help of two elementary schools located in disadvantaged neighbourhoods. It also discusses the results. In order to foster interest in homework, the Fouineur encourages the use of quality library resources and access to the specialists of the public library. To date, ten thousand children have participated in the workshops of the Fouineur.

\section{El Fouineur: un programa de ayuda al éxito} escolar de la ciudad de Repentigny

Basándose en el vínculo indisociable entre el acceso a los libros y el éxito escolar, este artículo presenta la creación de un programa de ayudá al éxito escolar establecido por la Biblioteca municipal de Repentigny, en los suburbios de Montreal. Refleja el proyecto piloto desarrollado en 2006-2007 en dos escuelas primarias situadas en barrios desfavorecidos y describe los resultados obtenidos con los años. Para incentivar en los jóvenes el gusto por los deberes, el Fouineur apuesta a la utilización de recursos documentales de calidad y a la disponibilidad de especialistas en la biblioteca municipal. Hasta la fecha, diez mil niños han participado en los talleres del Fouineur.

\section{Introduction}

$\mathrm{E}$ N 2005, L'ACTUALITÉ QUÉBÉCOISE faisait état d'un débat autour des besoins en matière d'aide aux devoirs dans les écoles primaires, des collections désuètes des bibliothèques scolaires, ainsi que des écarts entre filles et garçons quant aux habiletés de lecture. C'est aussi durant cette période que le ministère de l'Éducation, du Loisir et du Sport (MELS) annonçait son plan d'action sur la lecture à l'école ainsi qu'une subvention de 60 millions de dollars sur trois ans pour l'acquisition de livres et le soutien de la formation du personnel en animation de la lecture. Par ce plan d'action, le ministre Reid annonçait qu'il souhaitait faire découvrir aux jeunes le plaisir de lire en insistant sur le fait qu'il s'agissait du plus beau cadeau à leur faire, puisque la "réussite scolaire passe nécessairement par la lecture " (Québec. Ministère de l'Éducation, du Loisir et du Sport, 2005). Déjà, en 2003, un colloque de l'Association pour la promotion des services documentaires scolaires (APSDS) s'intitulant "La médiation de la lecture auprès des jeunes, le temps d'agir ! » avait établi une corrélation entre accès à la bibliothèque et réussite éducative des élèves et souligné l'importance du réseautage entre milieux scolaire et municipal dans l'atteinte d'un objectif conjoint de promotion de la lecture et du livre (Melançon, 2003).

À la Bibliothèque Robert-Lussier ${ }^{1}$ de la ville de Repentigny, en banlieue de Montréal, ce contexte nous interpellait beaucoup et nous étions persuadés que la bibliothèque municipale pouvait, sans se suppléer aux bibliothèques scolaires et en accord avec sa propre mission, intervenir concrètement auprès des jeunes de sa communauté. Cependant, nous constations avec consternation que malgré les liens évidents entre lecture et réussite scolaire, la salle jeunesse de la bibliothèque était quasi déserte après l'école, même si elle offrait plusieurs milliers de documents et une collection d'ouvrages de référence adaptée aux élèves du primaire. Nos jeunes citoyens n'avaient-ils pas besoin d'informations pour réaliser des recherches, d'outils pour faire leurs devoirs et d'un lieu calme et propice au travail scolaire? Ces jeunes que nous rejoignions si facilement la fin de semaine ou durant les vacances pour la lecture de détente ne nous visitaient guère dans le cadre

1. <http://www.ville.repentigny.qc.ca/bibliotheque/> 
de leur cheminement scolaire. Était-ce par méconnaissance des ressources disponibles? Parce qu'ils n'avaient pas de moyen de transport pour fréquenter la bibliothèque durant la semaine ? Ou simplement par manque d'intérêt?

Après analyse des statistiques de prêts, nous avons vite fait le constat que si nos collections documentaires pour jeunes étaient sous-utilisées pour répondre aux besoins scolaires, c'est que nous n'en faisions pas suffisamment la promotion. Il était devenu impératif pour nous de faire connaitre nos ressources et d'en montrer la pertinence dans le cadre scolaire.

Connaissant bien sûr la mission des bibliothèques publiques, qui inclut un volet éducatif, nous nous sommes documentés sur le lien entre l'accès aux livres et aux ressources documentaires plus généralement et la réussite scolaire. Sans faire ici une revue exhaustive des écrits, nous pouvons affirmer que non seulement ce lien ressortait clairement de nos lectures, mais encore, plusieurs chercheurs, dont Fryer et Levitt (2004), avançaient même qu'un jeune issu d'un milieu bien nanti et évoluant parmi les livres dès son plus jeune âge avait d'emblée plus de chances de réussite scolaire que les enfants du même âge ne bénéficiant pas d'un contexte aussi favorable ; Indramati (2010) a récemment confirmé cette observation. Les écrits montrent que certains facteurs de risques rendent les élèves issus de milieux défavorisés plus vulnérables; le soutien, la stimulation, la supervision ne font pas toujours partie de leur réalité familiale (Hart, 2010). Or, le plan d'action sur la lecture à l'école soutient que "si la passion pour la littérature est suscitée dès les premières années du primaire, il est fort probable que la lecture fera partie intégrante des passe-temps de l'élève et que cet attrait littéraire se maintiendra au cours de sa vie d'adulte" (Commission scolaire de Montréal, 2010). Ajoutons que le fait de lire à l'âge adulte a certainement des répercussions positives sur toutes les sphères de notre vie.

\section{Un projet-pilote}

Toujours en 2005, le Tableau de bord lanaudois sur la défavorisation (Leclerc et al., 2005) faisait ressortir les profils socio-économiques présents dans la Municipalité régionale de comté de L'Assomption. Nous avons utilisé ce tableau pour analyser plus particulièrement les données s'appliquant aux différents secteurs de Repentigny, car il devenait évident qu'il y avait une corrélation entre défavorisation et habiletés de lecture (Duke, 2000).

Repentigny, $12^{\mathrm{e}}$ ville en importance au Québec, était en 2005 une ville de 76237 habitants au revenu moyen plutôt élevé de $71737 \$^{2}$. Les résultats du Tableau

\footnotetext{
2. Données du recensement de 2001, région de Lanaudière. <www.stat.gouv.qc.ca/ regions/recens2001_14/revenu14/revstrucfam14.htm> (consulté sur Internet le 15 octobre 2010).
}

de bord illustrent clairement qu'il existe à Repentigny certains quartiers moins favorisés. Ces quartiers, bien identifiés et délimités par des cartes, sont en fait situés au pourtour de la Bibliothèque Robert-Lussier. On trouve deux écoles primaires dans cette enclave située à proximité de marche de la bibliothèque3. Forts de nos constats, nous avons rencontré les directions de ces écoles pour leur proposer un projet-pilote visant à faire découvrir la bibliothèque municipale et ses ressources à leurs élèves. Dès la première rencontre, les deux directeurs ont accepté l'offre avec enthousiasme ; les bases du programme Fouineur étaient jetées.

Ainsi, Le Fouineur serait d'abord un lieu où se trouveraient réunies les ressources pertinentes pour les élèves du primaire, et où ceux-ci pourraient bénéficier d'un climat propice au travail. Il s'agit là d'un beau souhait, mais comment obtenir le financement pour le concrétiser? Nous avons trouvé le partenaire financier tout indiqué en la Conférence régionale des élus (es) de Lanaudière. Le hasard faisant parfois bien les choses, il y avait, au moment de nos premières démarches, un appel de projets dans le cadre d'un fonds régional 4 visant à mettre en œuvre des programmes de lutte contre la défavorisation. Les élus de Lanaudière sont sensibles à tout ce qui touche la réussite scolaire, car dans notre région, malheureusement, les statistiques du décrochage scolaire sont supérieures à la moyenne provinciale. Les plus récentes statistiques font d'ailleurs ressortir l'écart: en 2007-2008, le taux de décrochage scolaire dans Lanaudière était de $32 \%$, comparativement à $25,3 \%$ pour l'ensemble du Québec 5 ; ce chiffre est à la hausse depuis 2004 (écart de 0,6\%).

Notre projet-pilote a été financé avec un budget total d'environ $40000 \$$, incluant la contribution de la Ville et celles de nos divers partenaires du milieu, dont la Conférence régionale des élus (es) de Lanaudière. Nous avons informé la Commission scolaire des Affluents de notre démarche et celle-ci, par l'entremise du directeur aux ressources didactiques, M. Fernand Lefebvre, a appuyé nos efforts en offrant des conseils pour faciliter le développement du programme et répondre aux préoccupations réelles des enseignants.

\section{Organisation du projet}

Nous avons en premier lieu défini notre objectif général. Que souhaitions-nous atteindre au final ? Et une réponse s'est imposée d'elle-même: que nos jeunes citoyens développent des habitudes de fréquentation assidue de leur bibliothèque municipale dans le cadre

3. Il s'agit des écoles primaires Pie-XII et Émile-Nelligan, de la Commission scolaire des Affluents.

4. Fonds de développement régional (FDR) : Entente spécifique sur le développement social, 2007.

5. Banque de données des statistiques officielles sur le Québec (BDSO). <http:// www.bdso.gouv.qc.ca/pls/ken/Ken263_Liste_Total.p_tratr_reslt?p_iden_ $\operatorname{tran}=$ REPERRE5F6V45-202133024735kH9:\&p_modi_url $=1116113630 \&$ p_id_ rapp $=1805 />$ (consultée sur Internet le 16 novembre 2010). 
Figure 1.

Les 3 volets du programme d'aide à la réussite scolaire Le Fouineur.

\begin{tabular}{|c|c|}
\hline $\begin{array}{l}\text { Le }{ }^{\text {er }} \text { volet : une animation en classe } \\
\text { Intervenant : animatrice }\end{array}$ & $\begin{array}{l}\text { Le } 2^{\mathrm{e}} \text { volet : visite des groupes scolaires à la } \\
\text { bibliothèque }\end{array}$ \\
\hline $\begin{array}{l}\text { En début d'année scolaire, une animatrice } \\
\text { (technicienne en documentation) de la }\end{array}$ & $\begin{array}{l}\text { Intervenant : bibliothécaire } \\
\text { professionnelle }\end{array}$ \\
\hline $\begin{array}{l}\text { bibliothèque se rend dans les classes pour } \\
\text { présenter Le Fouineur et ses ressources par } \\
\text { le moyen d'une animation ludique. }\end{array}$ & $\begin{array}{l}\text { Les groupes scolaires viennent travailler } \\
\text { au Fouineur durant les heures de classe. } \\
\text { Ils sont accueillis par une bibliothé- } \\
\text { caire professionnelle qui les initiera à la } \\
\text { recherche d'information en bibliothèque au } \\
\text { fil de leurs visites. }\end{array}$ \\
\hline
\end{tabular}

Le $3^{\mathrm{e}}$ volet : les ateliers de devoirs

Intervenantes en ressources pédagogiques (des enseignantes)

En fin d'après-midi, Le Fouineur devient un véritable supplément à l'école en offrant cette fois sur une base individuelle, des ateliers de devoirs dynamiques aux jeunes citoyens. Ces ateliers sont encadrés par des enseignantes. Deux enseignantes à la retraite sont employées par la Ville de Repentigny. de leur cheminement scolaire. Nous avions en tête un idéal : faire naître chez les jeunes le goût des livres et les inciter à utiliser les ressources de la bibliothèque pour faire leurs devoirs scolaires.

Un autre aspect de notre réflexion était de faire de l'espace Fouineur une destination en soi. Pour utiliser pleinement les ressources de la bibliothèque, il était primordial que les jeunes y passent un moment. Nous voulions développer un espace convivial où les jeunes se sentiraient à l'aise, un lieu qui serait à leur image et où ils seraient intéressés à revenir régulièrement. Nous voulions développer le plaisir de fureter parmi les livres, de les feuilleter, puis de sélectionner les plus pertinents pour le travail à réaliser. Mais comment espérer que les jeunes soient enthousiastes à l'idée de passer du temps à la bibliothèque municipale après avoir passé toute une journée à l'école? Et, par-dessous tout, comment leur communiquer le plaisir de venir y faire leurs devoirs ? Il fallait que notre offre soit vraiment attrayante et rassembleuse. Il était impératif que notre programme tranche avec l'école, qu'il ne dédouble pas les ressources déjà existantes et qu'il comporte une dimension ludique.

Nous savons que les jeunes ont un fort besoin d'appartenance et d'identification. Nous avons donc créé un personnage au look préadolescent, la fouine, pour le référent qui l'accompagne, l'idée de chercher pour tout savoir, de creuser pour obtenir plus d'informations. Les couleurs du personnage ont été choisies avec soin, car nous allions aussi les utiliser pour décorer l'espace Fouineur.

Nous disposions de peu de moyens financiers pour aménager l'espace. Il fallait un aménagement attrayant, coloré et qui invite à s'installer. Tout a été réalisé à l'interne. La graphiste de la Ville a créé le personnage et proposé un aménagement. Le menuisier de la Ville a conçu le mobilier à partir de matériaux recyclés. Nous avons tout misé sur les collections. C'est par les livres que nous voulions convaincre. Il s'agissait là d'un pari audacieux. La principale difficulté était que nous ne voulions pas nous contenter d'attirer les jeunes déjà lecteurs, mais prioritairement ceux qui ne lisaient pas et qui n'avaient pas encore découvert leur bibliothèque municipale.
Nous avons misé sur l'originalité. À la place d'étagères, le menuisier a construit des casiers rappelant ceux de l'école. Il les a peints d'un jaune fluorescent. Et comme nous nous adressions à un jeune public ayant peu ou pas d'expérience de la recherche documentaire, nous avons choisi de disposer la collection en nous basant sur la réalité scolaire, c'est-à-dire en regroupant les livres par matière.

Quelles étaient, exactement, les matières scolaires pour chacun des cycles du primaire? Quelles compétences étaient visées, quels savoirs devaient être acquis? Voilà des questions que nous nous sommes posées en plus de tenir compte de tous nos critères de choix habituels. Nous avons donc lu et relu les programmes du MELS. Nous avons examiné des revues spécialisées et les catalogues des éditeurs scolaires. Il était important que Le Fouineur soit complémentaire à l'école et qu'il ne dédouble pas l'aide existante. Ainsi, nous avons choisi de ne pas acquérir les manuels et cahiers d'activités utilisés comme matériel pédagogique. Nous avons plutôt opté pour "l'enrichissement " en regroupant, en plus des documentaires sur les matières scolaires, des ouvrages de référence visant spécifiquement les élèves du primaire, des collections de livres adaptés aux apprentis-lecteurs (et classés selon le niveau de difficulté), des revues éducatives, en plus de développer un secteur pour l'apprentissage de l'anglais et de l'espagnol. La ligne directrice de notre développement pour le coffre à outils du Fouineur se résume ainsi : chaque livre choisi doit être à la fois attrayant visuellement et attirant par son contenu pour un jeune.

Étant donné que la collection est notre principal agent motivateur pour insuffler le goût des devoirs, nous l'avons entièrement développée pour les besoins de la cause. Tous les documents qui en font partie sont neufs et récents. Nous l'avons baptisée le "Coffre à outils du Fouineur ».

Après réflexion, nous avons défini plus spécifiquement le projet Fouineur et c'est ainsi que nous l'avons présenté aux directions des écoles. 


\section{Un programme en trois volets}

Le programme comporte trois volets distincts qui visent à faire apprécier les ressources de la bibliothèque selon divers angles: livres pour le plaisir, pour la découverte, comme outil de travail. En plus des ressources documentaires, nous misons beaucoup sur les ressources humaines. La qualité du programme Fouineur est possible grâce à l'intervention des spécialistes qui sont tous des employés de la Ville de Repentigny. Ces spécialistes sont soutenus par une équipe de bénévoles.

En début d'année scolaire, l'offre de services Fouineur est transmise aux directeurs d'école. Après réception du dossier, plusieurs nous invitent à présenter ces services à l'équipe d'enseignants.

\section{Animation en classe}

Lors du projet-pilote, le premier contact avec les élèves s'est fait par une animation en classe. Les élèves d'une vingtaine de groupes des écoles primaires ÉmileNelligan et Pie-XII, soit 400 élèves environ, ont pris part à ces rencontres animées. C'est l'animatrice de la bibliothèque municipale qui se rendait en classe pour promouvoir le Fouineur et ses ressources. Nous nous sommes beaucoup questionnés sur l'orientation à donner à cette rencontre. Il nous fallait trouver une formule qui inciterait, à coup sûr, les enfants à visiter leur bibliothèque municipale.

Finalement, c'est par une présentation de livres judicieusement choisis pour chacun des niveaux que l'animatrice a su capter l'attention des élèves et leur a insufflé sa passion de la lecture. Et, bien sûr, les livres aux titres intrigants ont fait fureur. Voici des exemples de titres appréciés par les enfants: Toutes les réponses aux questions que vous ne vous êtes jamais posées ${ }^{6}$, Zarbi!: l'encyclopédie des animaux les plus bizarres 7 ou encore Dragonologie : encyclopédie des dragons ${ }^{8}$. Elle n'hésitait pas à relancer les élèves réagissant moins que leurs compagnons. Tu n'aimes pas lire? Et pourquoi pas un livre de blagues? Un recueil de statistiques sur le hockey? Après cette entrée en matière, elle distribuait à chacun une pochette à l'effigie du Fouineur contenant un formulaire d'abonnement à la bibliothèque, un dépliant à l'intention des parents et un signet pour les enfants. Le matériel promotionnel a été pensé de façon à assurer que ces documents soient lus. Ils sont de facture professionnelle, colorés, succincts et répondent bien aux questions du public visé. Par exemple, le document pour l'élève n'est pas un dépliant ou une lettre, mais plutôt

6. Nessmann, Philippe. 2006. Toutes les réponses aux questions que vous ne vous êtes jamais posées. 2. [Paris] : Palette, $128 \mathrm{p}$.

7. Zeitoun, Charline. 2005. Zarbi! : l'encyclopédie des animaux les plus bizarres. [Paris] : Mango jeunesse, $95 \mathrm{p}$.

8. Drake, Ernest. 2005. Dragonologie : encyclopédie des dragons. Toulouse : Milan jeunesse, $29 \mathrm{p}$. un signet illustré d'une bande dessinée l'informant qu'il peut faire ses devoirs au Fouineur après l'école. Cette formule promotionnelle a été très prisée par les élèves.

Le succès était au rendez-vous chaque fois que l'animatrice se rendait en classe pour présenter des livres. Nous avions alors plusieurs demandes de réservations pour ces mêmes titres le jour même. Il a fallu acheter les titres présentés en plusieurs exemplaires pour satisfaire la demande et ne pas risquer de perdre ces nouveaux abonnés. Cela représentait parfois jusqu'à cinq exemplaires par titre, ce qui est inhabituel pour la collection jeunesse.

Après que l'animatrice eût terminé la tournée des classes des deux écoles, les deux autres volets du programme se sont mis en place.

\section{Apprendre à chercher dans les livres}

Les enseignants ont la possibilité de travailler au Fouineur avec leurs groupes durant les heures de classe et de bénéficier des ressources réservées à leur intention. En plus du local et du Coffre à outils, nous préparons à l'intention des enseignants qui en font la demande de la documentation choisie sur des thématiques spécifiques. Les groupes scolaires sont accueillis par une bibliothécaire professionnelle qui les forme à la recherche d'information en bibliothèque au fil de leurs visites. Elle démystifie avec eux l'utilisation du catalogue, le fonctionnement de la classification, l'organisation d'une encyclopédie et elle les assiste dans leurs recherches documentaires. Une série d'ateliers visant l'intégration de ces notions spécifiques est présentée aux enseignants en début d'année.

Les enfants apprennent tout en s'amusant. Par exemple, pour les élèves de troisième année, la bibliothécaire a élaboré un jeu de rôles dans lequel l'enfant se fait éditeur et doit expliquer pourquoi le livre qu'il a choisi est réussi. Cette activité devient une introduction aux diverses parties du livre. Une activité de repérage dans les atlas apprend à l'enfant à repérer des pays et des villes en consultant l'index et en utilisant des indications telles que " $\mathrm{C} 9$, page 30 ». Ces devinettes incluent une notion de vitesse de réponse et gagnent vite l'enthousiasme des jeunes. La bibliothécaire coordonnatrice du Fouineur a développé une série de six ateliers qui sont proposés aux enseignants pour développer des habiletés spécifiques à l'aide de différents outils (atlas, encyclopédie, catalogue).

Pour faciliter l'intégration de ces nouvelles notions, un Guide d'initiation à la recherche est remis aux enfants à la fin de l'activité. Ce guide succinct et illustré explique la classification dans nos bibliothèques et comment faire une recherche au catalogue.

Les enseignants qui participent aux ateliers du Fouineur avec leur groupe peuvent utiliser la carte Fouineur, une carte-bibliothèque avantageuse leur allouant un nombre et une période d'emprunts augmentés. Ils 
peuvent emprunter une cinquantaine de documents pour une période de deux mois. Le but de ces privilèges est de leur permettre une transition simple du travail de recherche entamé au Fouineur et terminé en classe.

\section{Ateliers de devoirs ludo-éducatifs}

Le lieu dédié aux devoirs est garni de matériel éducatif amusant. Intervenantes et bibliothécaires ont acquis plusieurs ressources spécifiquement conçues pour aider aux devoirs. Par exemple, les enfants disposent d'une horloge pour apprendre l'heure, d'une pizza en pointes pour assimiler les fractions, de cartes-éclair pour pratiquer et mémoriser les tables arithmétiques, de cartes pour apprendre à bien tracer les lettres et les chiffres. Les intervenantes ont même développé du matériel lorsque celui-ci n'était pas disponible dans les commerces.

Les ateliers de devoirs se déroulent en dehors des heures de classe, soit du lundi au jeudi, de $15 \mathrm{~h} 30$ à $18 \mathrm{~h} 9$, et le dimanche de $13 \mathrm{~h}$ à $17 \mathrm{~h}$. À leur arrivée, les enfants s'inscrivent sur la feuille de présence, car nous tenons à comptabiliser certaines statistiques, dont le nombre de participants et les écoles qui ont profité de notre service. Mise à part l'inscription obligatoire à l'arrivée, l'heure des devoirs est libre. Ce sont les enfants eux-mêmes qui font appel à l'intervenante ou aux bénévoles pour obtenir de l'aide lorsqu'ils en ressentent le besoin. Les bénévoles agissent en «super parents ", c'est-à-dire qu'ils sont présents et dévoués, et entièrement à l'écoute des besoins des enfants puisqu'ils ne sont pas, comme les parents, distraits par la préparation du repas ou autre tâche familiale. L'équipe comprend six bénévoles choisis en fonction de leur intérêt pour les ateliers de devoirs, de leur expérience professionnelle et de leur disponibilité. Les bénévoles participent en début d'année scolaire à une demi-journée de formation animée par les intervenantes et la bibliothécaire. Au programme : explication du fonctionnement des ateliers de devoirs et des principaux types de demandes émanant des enfants, transmission de trucs et astuces, présentation des ressources documentaires et du matériel éducatif.

Pour fidéliser les jeunes aux ateliers de devoirs, nous avons eu l'idée d'une carte-récompense. Ainsi, chaque visite au Fouineur donne droit à un tampon. Après trois visites consécutives, l'enfant peut choisir une petite surprise (par exemple, un crayon ou une gomme à effacer). Nous avons adopté cette forme d'émulation à la suite de la proposition d'une enseignante. Ce qui compte pour l'enfant n'est pas tant le cadeau qu'il reçoit que le sentiment d'appartenance au groupe « sélect » des utilisateurs assidus du Fouineur; cela contribue certainement à sa motivation à fréquenter la bibliothèque. De plus, c'est un facteur de valorisation pour l'enfant que d'être accueilli à la bibliothèque par quelqu'un qui

9. Le lundi jusqu'à $17 \mathrm{~h}$, heure de fermeture de la bibliothèque connaît son nom et qui l'accompagne dans ses travaux et ses soucis scolaires au quotidien. Il s'agit d'un service à la clientèle sur mesure pour nos jeunes abonnés.

Bien entendu, il a toujours été possible pour les jeunes du primaire, à Repentigny comme ailleurs, de faire leurs devoirs à la bibliothèque municipale. Les ateliers de devoirs du Fouineur sont novateurs, parce qu'ils permettent à l'enfant qui les fréquente de bénéficier d'une nouvelle forme d'aide, complémentaire à celle qui existe déjà à l'école ou à la maison. Contrairement aux programmes d'aide des écoles, les ateliers de devoirs du Fouineur sont offerts à tous et non pas uniquement à ceux que l'on juge en avoir besoin. Ils visent davantage à stimuler et à motiver les enfants qu'à intervenir pour régler une difficulté spécifique. Les ressources documentaires permettent d'étayer le soutien apporté par l'intervenante qui peut ainsi développer chez les enfants le réflexe de chercher dans les livres pour valider une réponse ou un apprentissage. Lorsque les enfants doivent réaliser une recherche d'informations, l'intervenante les accompagne au bureau de référence pour que la bibliothécaire puisse poursuivre une recherche structurée avec eux.

\section{Cinq ans plus tard, des résultats tangibles}

\section{Reconnaissance du programme}

Dès sa création, Le Fouineur a attiré les jeunes et enthousiasmé leurs parents. Le bouche à oreille à lui seul a fait son œuvre, si bien qu'à la fin du projet-pilote, plusieurs écoles nous ont demandé de prendre part au programme. Certaines d'entre elles sollicitent même notre participation à la réunion de parents en septembre pour une présentation du programme. Nous croyons que le dévouement et le professionnalisme de l'équipe bibliothèque qui intervient auprès des jeunes ne sont pas étrangers au succès du programme.

Nous comptabilisons déjà plus de 10000 présences aux ateliers. Nous avons remporté en 2007 le prix Mérite Ovation municipale décerné par l'Union des municipalités du Québec, dans la catégorie développement communautaire, justement parce que nous avons fait ressortir le lien entre l'accès aux livres et la réussite scolaire. En 2009, le programme a reçu le Grand prix Desjardins de la culture pour la région de Lanaudière.

Le regroupement des bibliothèques publiques de Laval-Laurentides-Lanaudière a fait une demande au ministère de la Culture, des Communications et de la Condition féminine (MCCCF) pour que le programme Fouineur soit régionalisé. Ce projet ne peut être concrétisé pour le moment étant donné le budget nécessaire à l'exportation du concept pour l'ensemble de ces régions. Nous croyons que pour être gagnante, notre formule doit rester authentique. Ainsi, il faut conserver la combinaison « ressources documentaires - ressources 
humaines - aménagement ", ce qui implique des coûts importants. L'intérêt persiste néanmoins au sein de plusieurs municipalités. Nous sommes toujours à la recherche de financement.

Pour répondre à la demande grandissante, nous avons aménagé un deuxième espace Fouineur à la Bibliothèque Edmond-Archambault en 2008. Ainsi, les élèves du secteur Le Gardeur peuvent plus facilement profiter de ces services. Du côté de Repentigny, nous songeons à un agrandissement, car les enfants sont maintenant à l'étroit après l'école et ceci peut devenir rebutant. Certains enfants doivent même faire demitour parce qu'il n'y a plus de places libres à leur arrivée.

\section{Évaluation}

Contrairement à notre inquiétude de départ, le transport ne pose pas de problème pour la fréquentation des ateliers. Autant durant les heures de classe qu'après l'école, les enfants profitent du covoiturage avec des parents accompagnateurs. Nos registres de présences démontrent que des élèves en provenance des 18 écoles situées sur notre territoire, lequel s'étend sur 10 kilomètres, fréquentent Le Fouineur.

Nous appréhendions également que les ateliers de devoirs soient perçus par certains parents comme un service de garde gratuit et accessible à tous. Nous n'avons heureusement été que très rarement confrontés à cette situation. Les parents accompagnateurs s'installent pour travailler avec leur enfant, ou encore ils en profitent pour aller faire le plein de livres ou feuilleter des revues à l'espace café.

Les enfants qui ont pris part au programme Fouineur au fil des cinq dernières années ont été en contact avec un bibliothécaire de référence à de nombreuses reprises (promotion du programme, initiation à la bibliothèque, question de référence pendant les ateliers de devoirs). Ainsi, les jeunes Repentignois savent qu'il y a un professionnel à la bibliothèque municipale dont le travail est de les guider dans leurs recherches documentaires. Et plusieurs ont développé le réflexe de demander son aide puisque nos bibliothécaires remarquent qu'elles ont de plus en plus à effectuer des recherches à la demande d'enfants.

Bien entendu, malgré ce contexte positif, notre intervention a des limites. Lorsqu'un enfant a de sérieuses difficultés scolaires, nous ne sommes pas en mesure de l'aider. Nous informons le parent et le dirigeons vers des ressources plus appropriées. Et depuis un an environ, nos intervenantes sont aussi confrontées à une nouvelle réalité : celle des besoins spécifiques des nouveaux arrivants. Les jeunes immigrants ont découvert nos ateliers de devoirs, ce qui nous enchante particulièrement, sachant qu'il peut être difficile de rejoindre cette clientèle pour leur offrir des services adaptés. Cependant, ces jeunes ont parfois de nombreux besoins en matière d'aide aux devoirs, en lien par exemple avec l'apprentissage du français, et nous ne disposons pas de l'expertise qui nous permettrait de bien les accompagner. Or, communiquer cette réalité aux parents immigrants n'est pas toujours simple si ceux-ci ne maîtrisent pas eux-mêmes le français ou l'anglais. Pour remédier à cette situation, nous avons fait appel à un organisme de soutien aux familles immigrantes dans le but d'organiser en concertation une activité d'initiation aux ressources de la bibliothèque municipale à l'intention des nouveaux arrivants. Nous croyons que la bibliothèque municipale, et plus particulièrement Le Fouineur, est une porte d'entrée privilégiée pour faire connaissance avec une clientèle aux besoins particuliers et leur faire connaître nos ressources. Le Fouineur joue donc aussi un rôle de diffusion de l'information et d'intégration.

L'appréciation du programme a un impact en termes statistiques. Depuis la création du programme, le nombre d'enfants abonnés a augmenté de $26 \%$. Quant au sentiment d'appartenance, nous avons gagné notre pari puisque nous entendons maintenant nos jeunes abonnés dire: "Viens-tu faire tes devoirs au Fouineur?", comme si c'était une évidence, comme si tout le monde connaissait Le Fouineur.

\section{Sondage auprès des élèves}

Afin de mieux connaitre le niveau de satisfaction des usagers actuels du Fouineur, nous avons récemment mené un sondage auprès d'élèves qui fréquentent nos ateliers régulièrement. Soixante-quinze enfants ont accepté de répondre à notre questionnaire en classe ou sur place, dans l'espace Fouineur. Nous avons voulu savoir quelles étaient leurs sources de motivation pour fréquenter Le Fouineur. Ils les ont priorisées ainsi :

- Lire les livres de la bibliothèque ;

- Apprendre à chercher l'information ;

- Retrouver mes amis ;

- Faire mes devoirs ;

- Travailler en équipe.

Le premier constat est que la visite au Fouineur en groupe constitue souvent un premier contact avec la bibliothèque municipale. Trente pour cent des élèves ont répondu ainsi et cela rejoint la tendance observée par le MELS dans son analyse des habitudes et pratiques des élèves québécois de 10 ans (Rodrigue et al., 2009). D'ailleurs, en 2006, nous avions été surpris de constater cette réalité avec plusieurs élèves de l'école Émile-Nelligan dont le quartier de résidence est directement juxtaposé à la bibliothèque. Plusieurs d'entre eux n'avaient jamais encore mis les pieds à la bibliothèque. Nous avions alors dû revoir notre programme d'initiation à la recherche pour définir un atelier d'initiation à la bibliothèque de base qui présentait simplement ce nouvel environnement et la disponibilité des ressources aux élèves, par ailleurs ravis de leur découverte. 
Autre observation intéressante : les usagers les plus actifs sont en cinquième année et sont donc plus autonomes dans leur organisation du travail. Le deuxième groupe en importance est celui des premières années. Ceux-ci fréquentent habituellement l'espace Fouineur en famille dans un contexte d'apprentissage de la lecture. Les parents sont enchantés par la diversité et le nombre de ressources qui sont mises à leur disposition et ils apprécient l'effet motivateur de s'installer dans un lieu neutre pour travailler. Ainsi, ils peuvent se concentrer sur ce moment privilégié avec leur enfant, sans interférence des tâches ménagères, du téléphone ou de la télévision. Cela ne veut pas dire pour autant que l'espace Fouineur est silencieux. En présence de tous ces jeunes qui travaillent seuls ou en équipe, on perçoit un bourdonnement d'activités. Cela semble motivant pour les enfants. Il est à noter que la présence constante des intervenantes, en fonction depuis le début du programme, constitue un élément motivateur en soi. Dès 2006, nous nous étions rendus dans une classe de $3^{\mathrm{e}}$ année pour rencontrer les élèves et tourner une vidéo promotionnelle. Et à la même question («Pourquoi aimes-tu venir faire tes devoirs au Fouineur?»), nous avions alors résumé les réponses des élèves ainsi : pour les beaux livres, pour le silence (se traduisant par: "pas de télé », "pas de bébé qui pleure »), pour Madame Lorraine, notre intervenante en ressources pédagogiques. Cinq ans plus tard, ce sont les mêmes points qui ressortent. L'encadrement offert est apprécié par les jeunes. D'ailleurs, une vingtaine d'élèves ont souligné qu'ils fréquentent Le Fouineur pour l'aide qu'ils y reçoivent des adultes.

L'accueil personnalisé est un ingrédient essentiel à l'excellence du service à la clientèle. L'intervenante, présente depuis cinq ans aux ateliers de devoirs, personnalise nos services et ajoute au lieu une dimension humaine. À la question "Qu'est-ce que vous aimez le plus quand vous venez au Fouineur?", un enfant a coché toutes les cases, en plus d'ajouter « voir le sourire des adultes ». Effectivement, se sentir bienvenu semble avoir un impact indéniable auprès de nos jeunes fouineurs. Une étude menée récemment au Royaume-Uni pour le compte de Museums, Librairies \& Archives (MLA) reconnait que les éléments clés pour bien rejoindre la jeune clientèle dans un contexte d'apprentissage sont la qualité des ressources documentaires, un environnement stimulant et le contact amical avec le personnel (Ipsos MORI Social Reasearch Institute, 2010). Il se tisse aussi un lien intergénérationnel significatif entre les enfants et les bénévoles. Enfants et aînés profitent de cette relation privilégiée et développent un lien de confiance qui ajoute au climat favorable du Fouineur. Certains bénévoles œuvrent au Fouineur depuis ses débuts et connaissent tous les enfants par leur nom, y compris ceux qui reviennent les saluer une fois rendus au secondaire.
Parmi les commentaires spontanés des enfants, nous avons relevé quelques perles qui sont représentatives du sentiment général :

"L'aide des adultes est précieuse car des fois, au lieu d'avoir de la difficulté, nous apprenons et nous comprenons"

"Je suis bonne maintenant et intelligente. Bravo! Vous maidez beaucoup!"

"Le Fouineur est le meilleur endroit pour les devoirs/études, car nous avons plein de livres!"

D'autres éléments intéressants ressortent des sondages. Par exemple, certains enseignants mentionnent l'effet d'entraînement des enfants qui fréquentent Le Fouineur vis-à-vis de leurs parents. Une enseignante de l'école Émile-Nelligan décrit ainsi le Fouineur : "Le Fouineur est un petit coin de paradis pour nos élèves: calme, paisible et plein de ressources. Ça développe le goût de lire et l'habitude de fréquenter la bibliothèque. C'est un outil qui me supporte lors de mes différents projets. »

\section{De nouveaux projets}

\section{Le Fouineurt}

La Bibliothèque Edmond-Archambault (secteur Le Gardeur) est régie par un protocole d'entente entre la Ville de Repentigny, la Commission scolaire des Affluents et l'école secondaire l'Horizon. Cette bibliothèque, à double vocation municipale et scolaire, dessert au quotidien les élèves de l'école. Lorsque les ateliers de devoirs du Fouineur ont été mis en place à cette bibliothèque, des élèves de première secondaire ont pris l'habitude de s'y rendre après les cours, certains pour faire leurs travaux en équipe, d'autres pour demander de l'aide pour leurs recherches d'informations. Avec le temps, quelques-uns ont proposé leurs services pour venir en aide aux plus jeunes du primaire et ont décidé d'en faire leur projet de travail communautaire ${ }^{10}$ sous la supervision de l'intervenante.

Nous avons aimé l'idée d'utiliser les ressources du Fouineur pour faciliter l'intégration des jeunes de première secondaire. Cette localisation du Fouineur comprend donc une particularité : le coffre à outils est accompagné du Fouineur+. Le Fouineur+ a sa propre signature et regroupe des ressources ayant été choisies non seulement en fonction des matières scolaires, comme nous le faisons pour le primaire, mais en lien avec les objectifs des programmes d'études du secondaire. Évidemment, il n'est pas toujours simple de faire cohabiter les tout-petits qui apprennent à lire et les adolescents de première secondaire, autant d'un point

10. Il s'agit de l'Approche orientante du MELS. Les élèves de troisième secondaire doivent élaborer un projet personnel en vue de prendre connaissance d'un milieu de travail et de les préparer à leur insertion socioprofessionnelle. 


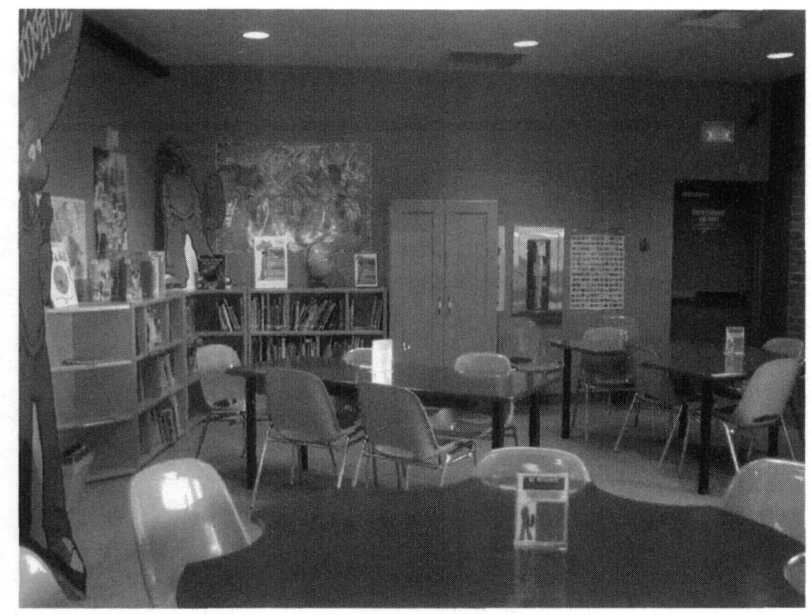

de vue social que par l'aménagement qui convient moins à ce dernier groupe d'âge. Un de nos objectifs est donc la subdivision des ressources en deux aires de travail différentes.

\section{Un lieu de diffusion}

Nos ateliers de devoirs comportent aussi une facette «animation». À la rentrée, nous offrons des conférences sur le thème de la réussite scolaire ou sur l'accompagnement des devoirs. Ces conférences sont données par des personnalités reconnues pour leur expertise. Nous prévoyons alors simultanément une activité à caractère pédagogique pour les enfants à même l'espace Fouineur. Par exemple, l'année dernière, nous avons offert aux parents la conférence de Louise Saint-Pierre, "Comment motiver les enfants à faire leurs devoirs", dans la salle d'animation, alors que les enfants profitaient des exercices de brain gym animés par le "Super héros des devoirs $A D$ " dans l'espace Fouineur. Par ailleurs, à certains moments stratégiques de l'année (avant les Fêtes, durant la semaine de relâche, en fin d'année scolaire), Le Fouineur fait relâche et c'est la fête pour tous les petits fouineurs assidus. Nous voulons récompenser leurs efforts soutenus et nous leur faisons parvenir une invitation à une activité d'animation spéciale (un spectacle, un film, un atelier avec un artiste) jumelée à un déjeuner. C'est l'occasion de venir passer du bon temps au Fouineur... et de bouquiner en famille.

\section{Difficultés liées au financement}

La seule ombre au tableau pour Le Fouineur est la précarité de son financement. Le maintien de ce programme coûte annuellement environ $40000 \$^{11}$. Il est clair que ce programme ne peut être absorbé à même le budget d'opérations d'une bibliothèque municipale, ni réalisé avec le seul personnel en place. Son succès réside dans la spécialisation des ressources, tant humaines que documentaires. Recevoir des groupes scolaires et leur

11. Ceci est la somme ajoutée au budget régulier. Si on inclut les services offerts et le personnel mobilisé, le total est plutôt de 65000 \$ par an.

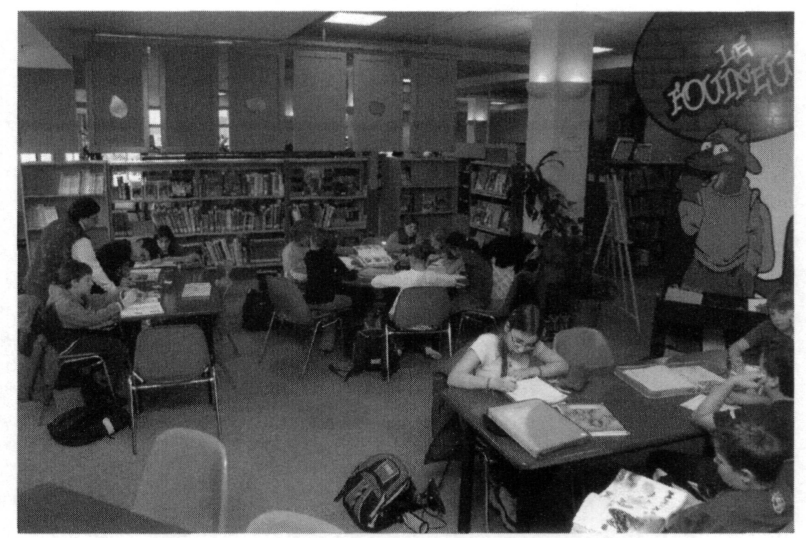

offrir des ateliers d'initiation à la recherche ou encore les encadrer dans leurs recherches, permettre le prêt en période de fermeture, tout cela nécessite du personnel additionnel et dédié à ces tâches. En ce qui a trait aux ateliers de devoirs, nous avons bien sûr mis en place des équipes de bénévoles, mais la stabilité du programme repose sur l'expertise, le professionnalisme et le leadership des intervenantes rémunérées. Maintenir en poste deux intervenantes en ressources pédagogiques 12 heures par semaine durant l'année scolaire, année après année, coûte cher et ne peut être justifiable sans subvention puisque nous ne sommes pas, à proprement parler, une institution d'enseignement. Depuis le début du programme, la Conférence des élus (es) de Lanaudière et le MCCCF ont soutenu financièrement le programme Fouineur. Les différents partenaires privés, sans grande visibilité à gagner pour leur contribution financière, ont financé ensemble plus de $20000 \$$. Comme toujours, le problème majeur est la récurrence des coûts. Nous réussissons à financer le projet, mais une année à la fois seulement. Nous ne sommes donc jamais assurés de la pérennité du programme. Cela implique que nos efforts sont, par la force des choses, axés sur le financement du programme plutôt que sur son développement. Il nous faudrait trouver un mécène, un partenaire majeur, qui deviendrait partie prenante de notre projet.

Une avenue déterminante aurait été un partenariat municipal/scolaire. Si les élèves bénéficient d'une d'aide complémentaire à celle fournie par les écoles grâce au Fouineur, les écoles profitent de services exclusifs et définis en fonction de leurs besoins réels. En 20072008, six écoles situées dans l'ouest de Repentigny ont contribué financièrement au programme Fouineur grâce au leadership d'un des directeurs, abonné assidu de la bibliothèque, qui croyait fermement aux retombées positives de nos ateliers de devoirs. Il jugeait que l'accès à nos ressources documentaires était un atout précieux pour les élèves de son école. Comme il y a beaucoup de mouvements de personnel au sein des directions d'école, la promotion du programme est sans cesse à refaire et une telle contribution financière est évidemment difficile à renouveler année après année. Les directions d'école, aussi enthousiastes soient-elles face au 
programme, ne sont pas convaincues du bien-fondé de leur participation financière à un projet municipal.

\section{Conclusion}

L'année 2011 constitue un heureux dénouement dans cette impasse financière. Nous sommes actuellement à élaborer une entente avec la direction de la Commission scolaire des Affluents qui a accepté de s'impliquer financièrement pour assurer la poursuite des activités du Fouineur auprès de l'ensemble des écoles de Repentigny. La Commission scolaire reconnait que notre initiative contribue à l'ensemble des efforts liés à la réussite scolaire. André Thibault, professeur-chercheur au Département d'études en loisirs, culture et tourisme à l'Université du Québec à Trois-Rivières, note que « aujourd'hui, il est urgent de concerter l'action pour faire face à [des] problématiques anciennes et nouvelles telles que le décrochage et la continuité des services. La concertation municipale/scolaire [...] agit bien au-delà de l'échange d'équipements et de locaux" (Thibault, 2008). Il présente le programme Fouineur comme un exemple concret de partenariat réussi avec le milieu. Même son de cloche du côté des directeurs d'écoles qui ont identifié le programme Fouineur comme moyen de formation des élèves à l'utilisation des ressources documentaires dans le Rapport d'évaluation du Plan d'action sur la lecture à l'école (Québec. Ministère de l'Éducation, du Loisir et du Sport, 2009).

Effectivement, en sollicitant la participation financière de la Commission scolaire, notre préoccupation va au-delà de la recherche de subvention. Notre souhait est plutôt de nous associer à ce joueur incontournable en matière de réussite scolaire et de nous en faire un allié, un partenaire. Par ailleurs, les recherches montrent que la coordination des interventions des bibliothécaires et des enseignants a des répercussions positives sur la réussite scolaire des élèves (Melançon, 2003). De cette collaboration résulte nécessairement un accès privilégié aux ressources documentaires en lien avec les apprentissages au quotidien. Donner accès aux ressources documentaires pour poursuivre, voire intégrer les apprentissages réalisés en classe, fait voir la bibliothèque comme "un prolongement naturel de la classe [...] » (Bougé, 2006). Idéalement, nous réussirons à former cette année un comité de travail Fouineur qui réunira à la même table bibliothécaires, animatrices, enseignants, directeurs d'écoles, parents. Cela, bien sûr, pour poursuivre l'évolution du programme et nous assurer de toujours le maintenir en lien avec les besoins réels de nos jeunes citoyens. Première retombée concrète de ce partenariat: dès le printemps prochain, une animatrice de la bibliothèque fera la tournée des cours d'écoles avec le nouveau chapiteau aux couleurs du Fouineur pour réaliser des journées d'animation de la lecture. Une récompense de fin d'année.

Nous avons encore plusieurs projets à réaliser avant que le Fouineur n'atteigne sa pleine maturité. Et des milliers de petits fouineurs à accompagner... Mais l'avenir du programme s'annonce prometteur grâce à notre nouveau partenaire. Plusieurs villes environnantes ont déjà manifesté leur intérêt à implanter le concept du Fouineur chez elles. $(-)$

\section{Sources consultées}

Bougé, R. 2006. Et toi, que lis-tu ? Bilan d'un riche colloque [organisé dans le cadre du Plan d'action sur la lecture à l'école]. Virage, $\mathrm{n}^{0} 1: 11$.

Commission scolaire de Montréal. 2010. Bilan du plan stratégique 2005-2010: Le défi de la réussite. En ligne $<\mathrm{http}: / / \mathrm{www} . c s d m$. qc.ca/CSDM/MissionEnjeux/PlanStrategique.aspx $>$ (consulté sur Internet le 3 mars 2011).

Duke, N.K. 2000. For the Rich It's Richer : Print Experiences and Environments Offered to Children in Very Low- and Very high-Socioeconomic Status First-Grade Classrooms. American Educational Research Journal, $\mathrm{n}^{\circ} 2: 441-478$.

Fortin, P. 2005. Des parents modèles? Le succès des enfants à l'école dépend de ce que les parents sont, pas de ce qu'ils font. L'Actualité, $\mathrm{n}^{\circ} 18: 84$.

Fryer, R.G. et Levitt, S.D. 2004. Understanding the Black-White Test Score Gap in the First Two Years of School. The Review of Economics and Statistics, $\mathrm{n}^{\circ} 2: 447-464$.

Hart, M. 2010. Pauvreté et défavorisation, de l'isolement social à la réussite scolaire. Vie pédagogique, $\mathrm{n}^{\mathrm{0}} 155$. En ligne <http://www.mels.gouv. qc.ca/sections/viepedagogique/155/index.asp ?page=dossierA_2> (consulté sur Internet le 16 novembre 2010).

Indramati, K.M.. 2010. Exploring educational material needs and resources for children living in poverty. En ligne $<\mathrm{http}$ :// www.eric.ed.gov/ERICWebPortal/detail ?accno=ED511948> (consulté sur Internet le 17 novembre 2010).

Ipsos MORI Social Reasearch Institute. 2010. What do the public want from libraries? En ligne <http://research.mla.gov.uk/ evidence/view-publication.php ? $\mathrm{dm}=\mathrm{nrm} \&$ pubid $=1161>$ (consulté sur Internet le 16 novembre 2010).

Leclerc, B.-S. et al. 2005. Tableau de bord lanaudois sur la défavorisation : territoire de Lanaudière : calibrage à l'échelle de la région de Lanaudière. Saint-Charles-Borromée : Service de surveillance, recherche et évaluation, Direction de santé publique et d'évaluation, 66-75.

Melançon, L. 2003. Il est minuit moins cinq, c'est le temps d'agir ! Lurelu, $\mathrm{n}^{\circ} 1$ : 99-101.

Poulin, A. 2004. Les bibliothèques colères. Lurelu, $\mathrm{n}^{0} 2:$ 5-9.

Québec, Ministère de l'Éducation, du Loisir et du Sport. 2005. Des mesures concrètes pour donner davantage le goût de la lecture aux jeunes du Québec. En ligne < http://www.mels.gouv.qc.ca/ CPRESS/cprss2005/c050114.htm/> (consulté sur Internet le 16 novembre 2010).

Québec. Ministère de l'Éducation, du Loisir et du Sport. 2009. Plan d'action sur la lecture à l'école : rapport d'évaluation. En ligne $<$ http://www.mels.gouv.qc.ca/sections/publications/publications/SICA/DRSI/EvalProg_PlanActionLectEcole_RapportEval.pdf> (consulté sur Internet le 16 novembre 2010).

Rodrigue, G. et al. 2009. Habitudes et pratiques des élèves québécois de 10 ans au regard de la lecture: document synthèse. [Québec] : Ministère de l'Éducation, du Loisir et du Sport. En ligne $<$ http://www.mels.gouv.qc.ca/sections/publications/publications/SICA/DRSI/QuEstCeQuOnLitA1oAns_08-01133.pdf> (consulté sur Internet le 16 novembre 2010).

Thibault, A. 2008. Le loisir public et civil au Québec. Montréal : Presses de l'Université du Québec, 165-166. 